\title{
A Framework to Support the Lifecycle of Virtual Manufacturing Enterprises
}

\author{
Américo Azevedo $^{1,2 *}$, Filipe Ferreira ${ }^{2}$, and José Faria ${ }^{1,2}$ \\ ${ }^{1}$ Faculdade de Engenharia, Universidade do Porto \\ Rua Dr. Roberto Frias 378 \\ ${ }^{2}$ Inesc TEC \\ 4200-465 Porto, Portugal \\ Campus da FEUP, Rua Dr. Roberto Frias 378 \\ 4200-465 Porto, Portugal
}

\begin{abstract}
This paper proposes a framework to support the full virtual manufacturing enterprise lifecycle from the creation to the operation and dissolution. The paper will show how the framework supports the activities involved in the setup of virtual manufacturing enterprise, how to run it and which tools are needed. Three main perspectives are considered: knowledge and information, methodology and Technology. A business case analysis is then presented in order to understand the framework applicability to the development of collaborative business opportunities in an engineer-to-order and one-of-a-kind production environments, which is common to a large number of SMEs.
\end{abstract}

\section{INTRODUCTION}

Nowadays, enterprises seek to adopt new business models and to explore new business organizations, such as dynamic networks and virtual enterprises, to quickly respond to market opportunities while assuring fast time to market, differentiated offerings and competitive prices [1]. Furthermore, business-manufacturing sustainability will be more and more found in high-variety and low-volume businesses associated with complex products manufacturing [2].

However, although several research initiatives and projects tried to address the needs and requirements of these emergent new business models, currently there are no proven or effective methodologies, approaches or tools to support the enterprises in creating, managing or participating in this type of new business organizations (e.g. dynamic networks and virtual enterprises) [3].

We understand "Virtual Manufacturing Enterprises" as one form of Collaborative Networked Organizations [6] that allows partners to exploit emerging business opportunities in a flexible way. Thus, taking into account this concept and in the context of Factory of Future initiative [11], the Adventure research project intends to design and develop a support infrastructure and to deliver a holistic environment for plug-and-play virtual factories and take into account the complete life cycle of these virtual manufacturing networks [4].

This paper presents a framework to support virtual manufacturing enterprises tackling the existing operational limitations by providing tools and practical approaches to leverage the exchange of information between factories at a manufacturing process level.

A business case analysis is presented in order to understand the framework applicability to the development of collaborative business opportunities in an engineer-to-order and one-of-a-kind production environments, which is common to a large number of SMEs.

\section{FOUNDATIONS AND RESEARCH TOPICS}

In today's industry, collaborative networks manifest in a large variety of forms. Moving from the classical supply chains format, characterized by relatively stable networks with well-defined roles and requiring only minimal coordination and information exchange, more dynamic structures are emerging in industry. Some of these organizational forms are goal-oriented, i.e. focused on a single project or business opportunity, such as the case of virtual enterprises [5].

Virtual enterprises have been widely discussed over the past decade. In this research work, the definition of virtual considered is based on the definition proposed by Camarinha-Matos [5]: A Virtual Enterprise represents a temporary

\footnotetext{
* Corresponding author: Tel.: (351) 22209 4000; E-mail: ala@fe.up.pt
} 
alliance of enterprises that come together to share skills or core competences and resources in order to better respond to business opportunities, and whose cooperation is supported by computer networks.

In the context of these research topics [6], several categories of goal oriented collaborative networks have been identified and defined and four of them are "grasping opportunity" driven and are naturally relevant in the scope of Adventure research project. This grasping opportunity driven Collaborative Networked Organizations are formed to answer a specific business opportunity and will dissolve once their mission is accomplished. Moreover, also relevant for the research work is the concept of Extended Enterprise.

An Extended Enterprise represents a concept typically applied to an organization in which a dominant enterprise "extends" its boundaries to all or some of its suppliers. An extended enterprise can be seen a particular case of virtual enterprise. This is typically the case of several SMEs in the manufacturing industry.

This paper is focused on an extended enterprise case in order to understand if the framework proposed fits the case requirements.

Several challenges are raised in context of virtual organizations. The integration of human and technical resources to enhance workforce performance and satisfaction; the need to instantaneously transform information gathered from a vast array of diverse sources into useful knowledge for making effective decisions; reconfigure manufacturing enterprises rapidly in response to changing needs and opportunities and the developing of innovative manufacturing processes and products with a focus on decreasing dimensional scale are some of the challenges pointes by researchers.

This represents two main challenges: (i) to find the right set of partners when a new business opportunity emerges and (ii) to integrate the legacy information systems so that cooperation can take place quickly and seamlessly.

\section{The Plug and Play Virtual Factory}

The concept of combining the power of several independent factories to achieve complex manufacturing processes as so-called virtual manufacturing enterprises is not new and has been addressed by several research projects in recent years [3][5][6]. However, most of them are limited to create virtual manufacturing enterprises at a business level and in many cases they concentrate on the partner-finding and factory-building processes. Still, no proven tools and technologies exist in the market to provide valuable end-to-end integrated Information and Communication Technology (ICT) in such environments [3]. Thus the current approaches do not allow for innovation and higher management and operational efficiency in networked operations and this projects aims to change this fact [4].

Several results obtained from ADVENTURE (ADaptive Virtual ENterprise ManufacTURing Environment) project will form the ADVENTURE framework allowing virtual manufacturing enterprises and enterprises move beyond existing operational limitations by providing concrete tools and approaches for leveraging the information exchange between factories [4].

\subsection{TECHNICAL APPROACH}

ADVENTURE is addressing the integration, interoperability, and collaboration challenges for SMEs in the manufacturing industry. Based on the paradigm of Service-oriented Architectures (SOA), exploiting latest technologies from the "Internet of Things", and leveraging recent developments in the area of Cloud computing, ADVENTURE will provide SMEs with tools and methods to tackle the challenges described in Section 2 by improving information exchange with other SMEs within virtual manufacturing enterprises throughout dynamic manufacturing processes [4].

SOA manufacturing approach enables the description of single manufacturing process steps as services [7][8]. Thus, ADVENTURE will enable SMEs to model their manufacturing processes as a composition of services.

ADVENTURE will support SMEs in ad hoc participating in (large) supplier/ customer networks. It will provide SMEs with tools and methods to design, simulate, optimise, execute, adapt and monitor dynamic manufacturing processes through the ADVENTURE web based dashboard (see Fig.1) and thus lower market access barriers for SMEs, which usually neither have the capabilities to control the whole manufacturing lifecycle nor the market power to enforce the own interfaces and standards. 


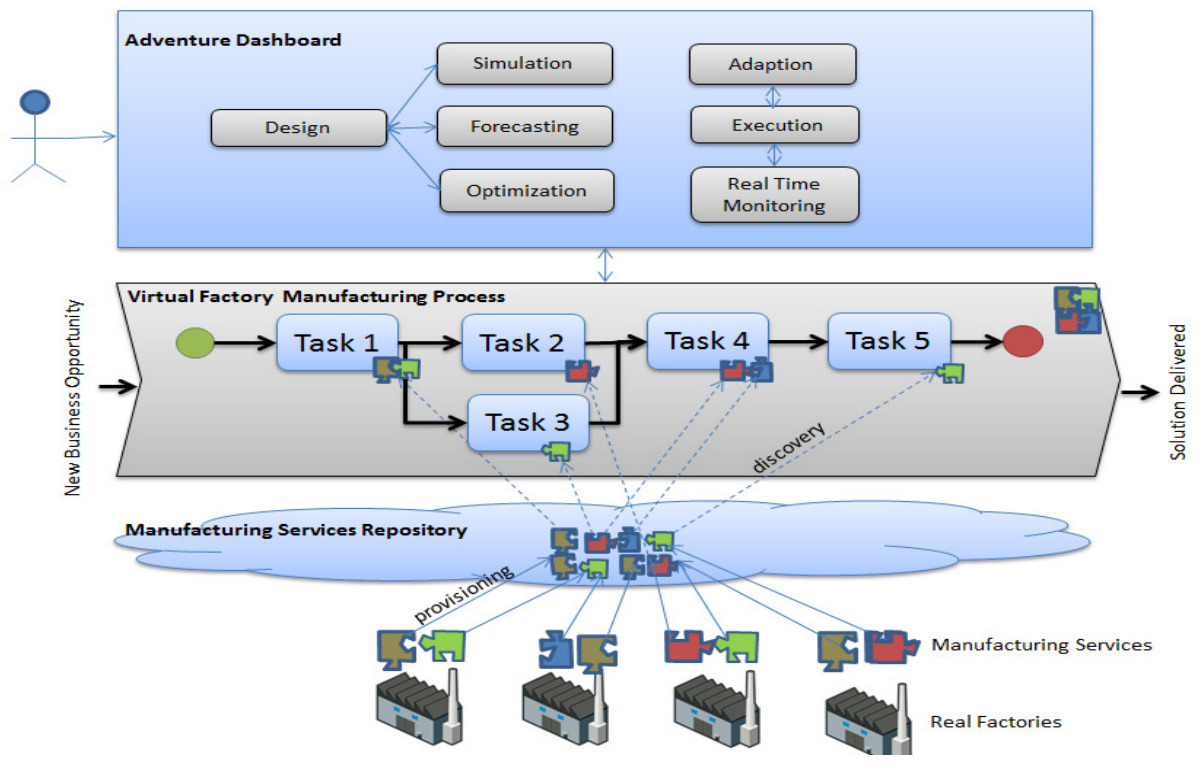

Figure 1. Adventure Vision.

\subsection{ENABLING FRAMEWORK}

Several results enabling ADVENTURE's vision are expected from the project. These are not only limited to various software components, but also include a knowledge and information repository and a methodology. The results will form the ADVENTURE Framework (Figure 2).

ADVENTURE defines the Virtual manufacturing enterprise methodology, which encompasses five main phases: Process Analysis, Design, Execution and Adaptation, Improvement and Dissolution as shown in Figure 3.

The process analysis phase refers to a preliminary business opportunity analysis in order to decide, whether an opportunity is reasonable and profitable or not. In this phase, the objectives, boundaries, inputs, outputs and stakeholders of the potential processes as well as the performance measures and recommendations are defined and developed, respectively.

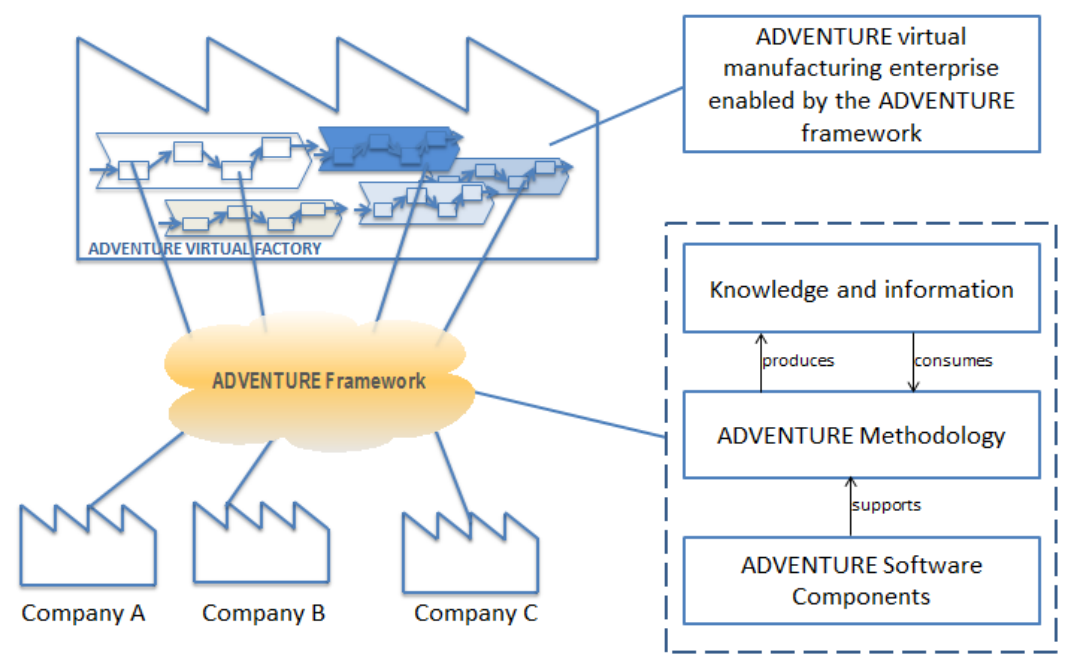

Figure 2. Adventure Framework. 
The design phase includes tasks of process definition, partner search and assignment, process simulation and process optimization. The user is able to define a new virtual manufacturing process using the smart process definition tool. Then, the partners to be assigned to each of the process activities can be searched by using the Data Provisioning and Discovery module. Once partners have been assigned to the manufacturing process activities, the process simulation plug-in can be used in order to obtain the results of a given process configuration. If the results are not satisfactory, it is possible to use the optimization component to achieve best global results for the manufacturing process. Thus, this component, applying linear programing techniques and other optimization models, allows the optimization of partner services assignment satisfying constraints and minimizing or maximizing the defined objective function.

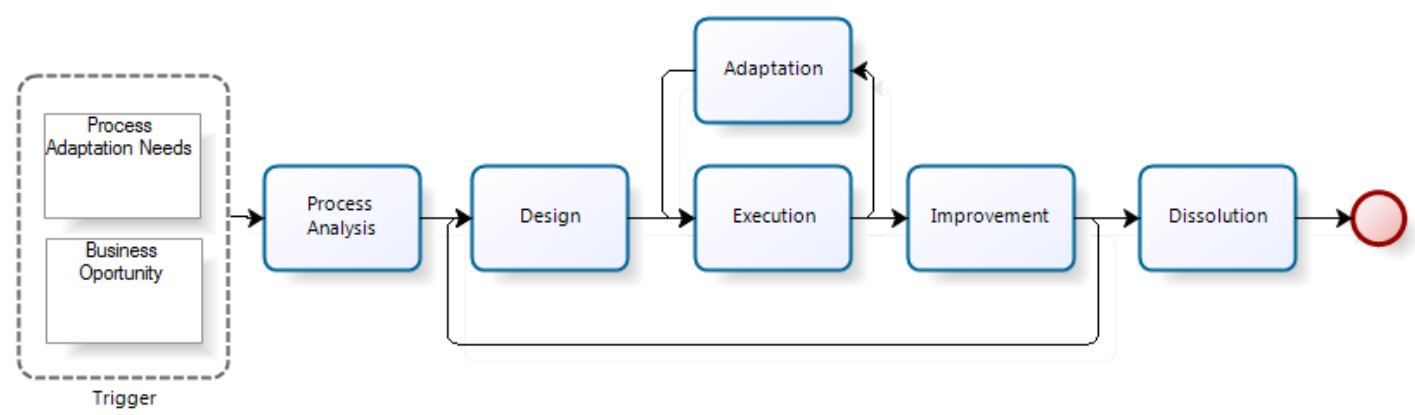

Figure 3. ADVENTURE Virtual manufacturing enterprise lifecycle main phases.

The execution and adaptation phases include tasks of process execution, monitoring, and adaptation. When the process is triggered, a workflow engine will manage the sequence of activities and invoke the associated manufacturing services. The monitoring module is then used to get in-depth information regarding relevant process task data, this module will include a rules engine and will be responsible for triggering alerts and notifications. Information from legacy systems and wireless sensors will be available by means of a technical integration. This way a real time process monitoring is achieved.

The improvement and dissolution phases refer to evaluating the executed process and members inquiry. Within ADVENTURE all the achievements (information, knowledge and experience) are stored in the cloud base data storage - the ADVENTURE Knowledge and Information Repository - which enables future ADVENTURE Virtual manufacturing enterprises to profit from this enhanced knowledge base.

It should be noted that will have a relevant role in the Adventure framework the set of reference business processes that will be used as a basis for the definition of the different processes operationalizing the business model of Virtual Manufacturing Enterprises. In fact, the concept of business process is becoming increasingly important and has been widely accepted as central to an organization's operations, namely to understand how it creates value for its customers. In this paper we adopt the definition of Weske [10] for business process: "A business process consists of a set of activities that are performed in coordination in an organizational and technical environment. These activities jointly realize a business goal".

The study already been made around some industry or domain-specific areas as well as the domain requirements analysis performed, allows to conclude that some available frameworks (e.g. SCOR [12] and VRM [13]) will be of significant potential as a foundation of the Adventure Reference Model (ARM) [4]. Furthermore, we are also looking for other business processes reference frameworks, namely the ones resulted from research projects in same or close research domains (e.g NetChalleng project [3]). 


\section{BUSINESS CASE}

One of the Business cases studied in the context of ADVENTURE is characterized by Complex products, one-of-a-kind environment and multiple business partners' involvement. Business needs and requirements have been gathered in order to understand the applicability of the envisioned framework to this kind of environment.

\subsection{BUSINESS CASE NEEDS}

In this kind of environment, for each customer order, there are specific engineering and production activities which should be managed as a whole. For each customer order, there is a mix of internal and external orders. Internal orders are usually managed by existing legacy systems, but the external orders are not. Both engineering and production activities could be outsourced (in each customer order, several activities could be outsourced).

Those business processes are non-prescriptive. Thus, there is an initial rough process design for each instance; followed by a progressive process refining based on human decision (human-based flexible and adaptive processes) [9].

A distinctive feature of those types of virtual manufacturing enterprises lies in the fact that they involve product design, process engineering and manufacturing tasks. All these tasks have to be managed comprehensively by the virtual manufacturing enterprise management system.

In such an engineer-to-order virtual manufacturing enterprise, several documents are created in execution time. For each customer order, specific documentation is generated for engineering, production, quality and after-sales activities. This documentation is spread on different systems and supports (e.g. paper, electronic, CAD, text processor office files).

Currently, there is no solution that meets mainly the functional requirements for this type of environment. The collaborative manufacturing process is managed ad-hoc and although partners are chosen on the basis of the qualification process, when it is necessary to collaborate, partners are bilaterally contacted. The whole process is managed manually using email exchange, phone calls and physical meetings.

In fact, there is a process where the human decision factor is very strong and where information is not correctly managed. Typically, the project manager knows the status of each task by direct contact with the responsible partners. Much information is kept in peoples' minds and never gets to be registered. This gives rise to errors and delays in the delivery of the final product.

\subsection{FRAMEWORK APPLICATION}

According to the ADVENTURE Virtual manufacturing enterprise lifecycle, respective activities of its main phases can be performed in order to meet the business case requirements.

Process Analysis phase. During process analysis phase, the new business opportunity and the process adaptation needs are examined in order to be able to create an abstract model of the Manufacturing Process for the envisioned ADVENTURE Virtual manufacturing enterprise. The objectives, boundaries, inputs, outputs, and stakeholders of the potential manufacturing Processes are defined as well as the performance measures and recommendations. To support this activity, ADVENTURE provides the Adventure Reference Model (ARM), consisting on a business model development method, a process catalog and a set of practices. These guidelines will be available in the Knowledge and Information Repository. Moreover, it is possible to search and query it in order to obtain information about similar processes that have been executed in the past. Using the ADVENTURE partner search engine, managers are able to search partners and filter them by industry type, location, work load level, etc. Furthermore, some preliminary simulations can be done in order to decide whether an opportunity is reasonable and profitable or not.

At the end of this phase, broker has gathered all the information necessary to decide if the collaborative manufacturing process should be implemented or not.

Design phase. During design phase, the mentioned abstract model of the Smart Process is developed, which describes the whole envisioned manufacturing process, and the therefore required services in terms of resources and manufacturing capabilities, which are offered by real factories.

Using the Smart Process Definition component, the Manufacturing Processes are designed following a service-oriented approach, including constraints as, e.g., environmental and ethical questions, lead time, and costs. Partners search is on manufacturing capability descriptions, available in ADVENTURE's Knowledge and Information 
Repository, including, e.g., delivery time, quality and technical requirements. Partners are then assigned to each of the process activities or sub-processes.

Once the process is completely defined, the simulation component can be used in order to evaluate whether all requirements are satisfied. Otherwise, optimisation techniques to the modelled Manufacturing Processes can be applied in order to make sure that the requirements are met and an efficient process execution is achieved. These optimisation techniques include support for achieving an optimised selection and assignment of partners to respective process steps as well as propositions for rearranging and restructuring the modelled Manufacturing Processes.

If all requirements are fulfilled during the simulation and forecasting, it is possible to start negotiating contracts with eligible candidate partner factories in order to achieve the best results.

Execution and Adaptation phase. During the execution and adaptation phase, the designed Manufacturing Process will be executed and adapted if needed. The best-fitting and contracted partner factories, identified and selected during the design phase, are employed and the modelled Manufacturing Process is executed and monitored.

While the actual execution takes place, ADVENTURE's monitoring component collects and provides real-time information about the current status of the Manufacturing Process. Unforeseen events may either be recognised by this monitoring component or by (intermediate) products or product parts enhanced with monitoring and error detection capabilities. Information from the different ICT systems and sensors of the customers, suppliers and shipment partners as well as from human input is also integrated into process monitoring.

The process status information will be prepared in such a way that there will be no difference between executing and monitoring a company-internal or cross-organisational manufacturing process.

If the monitoring shows unforeseen events which might change the outcome of the process with regard to deadlines, costs or other criteria, of if something different goes wrong with the contracted suppliers during the process runtime, an automatic alert indicating necessary adaptations of the process will take place, e.g., for redirecting distinct manufacturing steps to other partners or for exchanging underperforming, unsatisfying partners. The selection of alternative or complementary candidate factories and their manufacturing services, respectively, can either be performed manually or can be automatically assisted and performed by ADVENTURE's Process Optimisation component, which automatically composes a list of best matching partners and proposes this list to the process manager. The system thereby lets process manager take the final decision or automatically selects the best fitting suppliers depending on his preferences.

Also if requirements of the process change, e.g., because more products are needed or different features of a product are mandated, the process adaptation or optimization option as described above will be presented automatically.

Improvement phase. When an ADVENTURE Virtual manufacturing enterprise lifecycle reaches its end, an evaluation is taking place in the improvement phase. For this, process manager agrees with the partners, who have participated in this ADVENTURE Virtual manufacturing enterprise, on evaluating the executed Process. The reference models can be improved and lessons learnt are stored in the ADVENTURE Knowledge and Information Repository. ADVENTURE Members can access it in the future. This knowledge and information thereby is owned and maintained by the ADVENTURE Members.

Thus, in the improvement phase, information from the virtual manufacturing enterprises such as best practices and lessons learnt are created (and stored) so that an ADVENTURE Broker can query the Knowledge and Information Repository in order to obtain information about Manufacturing Processes that have been executed in the past, (probably) applying retrieved practices and avoiding mistakes which have been identified. This way, ADVENTURE builds a knowledge base which is enriched and updated each time a Process is executed.

Dissolution phase. After having updated the ADVENTURE Knowledge and Information Repository, the ADVENTURE Virtual manufacturing enterprise finally is dissolved. However, as all the information and configurations of the dissolved virtual manufacturing enterprise have been stored, it is possible to re-initiate the virtual manufacturing enterprise - conducting adaptations based on lessons learnt which have been stored during former improvement phases - in case, the same product has to be manufactured again. 


\section{CONCLUSIONS AND FURTHER RESEARCH}

The reality in today's business is to compete with dynamic and shorter market opportunities. In order to cope up with such environment, manufacturing firms, especially small and medium size enterprises (SMEs) need to collaborate with each other for mutual benefits. In that context emerges the concept of Virtual Enterprises.

The execution of virtual enterprise can be compared with the concept of 'Plug-and-Play' strategy, where various business processes are designed, validated and adopted according to specific need. During plug phase, specific business requirements those are collected previously are integrated with the process design phase. Whereas, in the play phase, predefined processes are adapted with the objective to run the Virtual Enterprise successfully.

The ADVENTURE framework aims to provide SMEs with the tools and methods to simplify the establishment, management, adaptation, and monitoring of dynamic manufacturing processes in virtual manufacturing enterprises by building on concepts and methods from the field of Service-oriented Computing.

The business case presented shows that it fits almost all the requirements needed to manage those collaborative manufacturing processes. However, to meet some specific requirements of a one-of-a-kind and engineer to order production environment, some improvements should be addressed, namely integration with content management systems and human based decision. Furthermore, as next steps, we intend to validate the ADVENTURE framework in different domain applications grounded in adaptive collaborative manufacturing environments.

\section{ACKNOWLEDGEMENTS}

This work is co-financed by the North Portugal Regional Operational Programme (ON.2 - O Novo Norte), under the National Strategic Reference Framework (NSRF), through the European Regional Development Fund (ERDF) and by the Portuguese Government through FCT - Foundation for Science and Technology.

\section{REFERENCES}

[1] Jovane, F., Westkamper. E., Williams, D.: The Manufuture Road: Towards Competitive and Sustainable High-Adding-Value Manufacturing. Berlin Heidelberg: Springer-Verlag, 2009.

[2] Azevedo, A., Almeida, A.: Factory Templates for Digital Factories Framework. Robotics and Computer-Integrated Manufacturing, 27, 755-771, 2011.

[3] Luis Carneiro et al.: An innovative framework supporting SME networks for complex product manufacturing. Collaborative networks for a sustainable world, Volume 336, p. 204-211, 2010.

[4] ADVENTURE: D2.1 Project Vision Consensus Document. Deliverable D.2.1 of Adventure Project - ADaptive Virtual ENterprise ManufacTURing Environment FoF-ICT-2011.7.3 - 285220, 2011.

[5] Camarinha-Matos, L.M., et al.: Collaborative networked organizations - Concepts and practice in manufacturing enterprises. Computers \& Industrial Engineering, 2009.

[6] Camarinha-Matos, L.M., Afsarmanesh, H.: Collaborative networks-Reference modeling. Springer, New York, 2008.

[7] Adams, M., et. al.: Worklets: A Service-Oriented Implementation of Dynamic Flexibility in Workflows. In: Proceedings of 14th International Conference on Cooperative Information Systems (CoopIS'06), pp. 291-308 Springer, 2006.

[8] Barba-Sánchez, V., Martínez-Ruiz, M.P., Jimenez-Zarco, A.I.: Drivers, benefits and challenges of ICT adoption by small and medium sized enterprises (SMEs): a literature review. Problems and Perspectives in Management, 5(1):104-115, 2007.

[9] Faria, J., Silva, J., Marques, H.: Supporting the Operation of Semi-Structured Work Systems, Proceeding of CENTERIS 2010 - Conference on Enterprise Information Systems, Viana do Castelo - Portugal, 2010.

[10] Wesk, M.: Business Process Management - concepts, languages, architectures. Springer, 2007.

[11] FoF-PPP: Factories of the Future: Public, Private Partnership, Strategic Multi-annual Roadmap. www.manufuture-industry.org, 2009.

[12] SCC: SCOR - Supply-Chain Operations Reference Model. Supply-Chain Council, 2011.

[13] VCG: VRM - Value Reference Model. Value Chain Group, 2010. 